\title{
植物根尖细胞的类角蛋白中间纤维 ${ }^{*}$
}

\author{
邢力 黄景花 扬 澄 \\ （北京大学生物系, 北京 100871)
}

\section{关镜词琓互根尖细胞、中间纤维、角蛋白、选择性抽提、免疫印迹法}

中间纤维一直被认为是广泛存在于动物细胞中的一类细胞骨架系统, 它的直径是 $10 \mathrm{~nm}$. 那么在植物细胞中是否存在中间纤维? 人们曾从多方面做过探索, 但至今还是一个没有解决 的问题. Dawson (1985) 和 Miller (1985) 用免疫化学的方法初步证明在洋葱根尖细胞和衣 藻（Alga Chlamydomonas） 中有类似动物细胞中间纤维的抗原物质存在 ${ }^{[1,2]}$. Powell (1982) 从悬浮培养的胡夢卜原生质体中能观察到抗非离子去垢剂和高盐溶液的 $7 \mathrm{~nm}$ 纤维 ${ }^{[3]}$. Goodbody（1989）虽证明中间纤维抗体可同悬浮培养的胡夢卜原生质体发生阳性反应, 但免疫金 颗粒都是结合在微管上 ${ }^{[4]}$. 因此以上这些工作都没有说明植物细胞内是否存在 $10 \mathrm{~nm}$ 中间纤 维, 更没有说明其蛋白质成分是什么.

苏菲、翟中和 $(1989 、 1990)$ 用选择性抽提技术首次证明在玉米和烟草叶肉细胞中存在类似 动物细胞中间纤维的网状结构, 这些纤维与动物细胞角蛋白抗体有阳性反应 ${ }^{[5,6]}$. 为了进一 步证明植物细胞中间纤维是否确切存在, 以及类角蛋白中间纤维是否在植物各种组织的细胞 内广泛存在, 我们主要选用䇋豆根尖细胞作材料进行了研究, 并以黄豆、叠豆根尖细胞和白 菜、峑豆叶肉细胞作对比试验, 不仅证明植物叶肉细胞内含有类角蛋白纤维, 而且首次证明在 植物根尖细胞内也存在 $10 \mathrm{~nm}$ 的纤维, 并含有二种类角蛋白, 其分子量为 52 和 $64 \mathrm{kD}$.

\section{一、材料及方法}

1. 原生质体的分呙挑选豌豆种子 50 粒在室温下黑暗的条件中萌发二天后, 取根 尖 $1 \mathrm{~cm}$, 横切成 $0.5-1.0 \mathrm{~mm}$ 厚的薄片, 在含有 $3 \%$ 的纤维素酶 (Onozuka R-10) 和 $0.5 \%$ 的 离析酶 (Yakult R-10) 的 $\mathrm{CPW}_{13}$ 溶液中酶解 $\left(\mathrm{CPW}_{13}\right.$ 溶液: $13 \%(W / V)$ 甘露醇、 $\mathrm{KH}_{2} \mathrm{PO}_{4}$ $27.2 \mathrm{mg} / \mathrm{L} 、 \mathrm{KNO}_{3} 101 \mathrm{mg} / \mathrm{L} 、 \mathrm{CaCl}_{2} \cdot 2 \mathrm{H}_{2} \mathrm{O} 1480 \mathrm{mg} / \mathrm{L} 、 \mathrm{MgSO} 4 \cdot 7 \mathrm{H}_{2} \mathrm{O} 240 \mathrm{mg} / \mathrm{L} 、 \mathrm{KI} 0.16$ $\left.\mathrm{mg} / \mathrm{L} 、 \mathrm{CuSO}_{4} \cdot 5 \mathrm{H}_{2} \mathrm{O} 0.025 \mathrm{mg} / \mathrm{L}, \mathrm{pH} 5.6\right) ， 30^{\circ} \mathrm{C}$ 条件下用摇休振荡3一 $4 \mathrm{~h}$, 胞壁被酶解后用 200 目尼龙网过滤, $1500 \mathrm{r} / \mathrm{min}$ 离心 $5 \mathrm{~min}$ 沉淀原生质体, 用 $\mathrm{CPW}_{13}$ 溶液冲洗二次, 此时原生 质体在光镜下呈均匀的圆球状.

取白菜或素豆真叶数片,撕去下表皮后放人 $15 \mathrm{ml}$ 含 $0.5 \%$ 纤维素酶及 $0.5 \%$ 离析酶的培 羊液中(含 $4.55 \%(W / V)$ 山梨醇、 $4.55 \%(W / V)$ 甘簬醇、 $0.5 \%(W / V) \mathrm{CaCl}_{2} \cdot 2 \mathrm{H}_{2} \mathrm{O}, \mathrm{pH}$. 2 ), $30^{\circ} \mathrm{C}$ 条件下酶解 3-4h. 胞壁被酶解后用 200 目尼龙网过滤, 500 $/ \mathrm{min}$ 离心沉淀原生质体, 用培养液洗原生质体二次, 镜检可见原生质体呈球形, 叶绿体均匀分布.

2. 细胞成分的选择性抽提原生质体用含 $0.5 \%$ Triton X-100 的 CSK 溶液轻轻吹

本文 1990 年 7 月 31 日收到.

- 国家自然科学盖会虫助项目. 
散, 静置 3-5min (CSK 液: $10 \mathrm{mmol} / \mathrm{L}$ PIPES、300 mmol/L 蔗糖、100 $\mathrm{mmol} / \mathrm{L} \mathrm{KCl} 、 3 \mathrm{mmol} / \mathrm{L}$ $\left.\mathrm{Mg} \mathrm{Cl}_{2} \cdot 6 \mathrm{H}_{2} \mathrm{O} 、 1.2 \mathrm{mmol} / \mathrm{L} \mathrm{PMSF}, \mathrm{pH} 6.8\right)$, 溶去原生质体膜和大部分可溶性蛋白质, 1000 $r / \mathrm{min}$ 离心 $5 \mathrm{~min}$, 弃去上清液, 沉淀用 RSB-Magik 溶液吹散, 静置 5-7 min (RSB-Magik 溶 液: $42.5 \mathrm{mmol} / \mathrm{L}$ Tris、 $8.5 \mathrm{mmol} / \mathrm{L} \mathrm{NaCl} 、 2.6 \mathrm{mmol} / \mathrm{Mg} \mathrm{Cl}_{2} \cdot 6 \mathrm{H}_{2} \mathrm{O} 、 1.2 \mathrm{mmol} / \mathrm{L} \mathrm{PMSF} 、 1 \%$ $\mathrm{Tween}-40 、 0.5 \%$ 脱氧胆酸钠, $\mathrm{pH} 8.3$ ), 此步骤可以去掉微管、微丝和其它一些结构蛋白质, 此 时胞质内主要存留中间纤维, $1000 \mathrm{r} / \mathrm{min}$ 离心 $5 \mathrm{~min}$ 后保留沉淀. 以上操作均在 $4^{\circ} \mathrm{C}$ 条件下 进行. 上述沉腚用含 DNase I $(100 \mu \mathrm{g} / \mathrm{ml})$ 的 DB 溶液室温下消化 $20 \mathrm{~min}$ (DB 溶液: 50 $\mathrm{mmol} / \mathrm{L} \mathrm{NaCl} 、 300 \mathrm{mmol} / \mathrm{L}$ 蔗糖、100mmol/L PIPES、3mmol $/ \mathrm{L} \mathrm{Mg} \mathrm{Cl}_{2} \cdot 6 \mathrm{H}_{2} \mathrm{O} 、 1 \mathrm{mmol} / \mathrm{L}$ EGTA, pH6.8), 使细胞核内染色体解旋, 然后滴加 $1 \mathrm{~mol} / \mathrm{L}\left(\mathrm{NH}_{4}\right)_{2} \mathrm{SO}_{4}$ 至终浓度为 $0.25 \mathrm{~mol} / \mathrm{L}$, $4^{\circ} \mathrm{C}$ 下静置 $3 \mathrm{~min}, 1500 \mathrm{r} / \mathrm{min}$ 离心 $10 \mathrm{~min}$ 后弃去上清液、核内碱性蛋白质随之消失, 最终取 得的样品主要是中间纤维与核内骨架体系.

以上步骧主要参照 Penman 的方法, 并稍加修改 ${ }^{[7,8]}$.

3. 装样品创各有植物原生质体的悬浮液, 滴在涂有 poly lysine 的 Formar 膜 復盖的铜网上, $30^{\circ} \mathrm{C}$ 静置 $20 \mathrm{~min}$ 后, 基本按上述步骤分级抽提, 只是处理时间稍短, 且不需要 离心. 经 CSK 漂洗后, 用 $2.5 \%$ 的戊二酫-CSK 固定 $60 \mathrm{~min}, 1 \% \mathrm{OsO}_{4}-\mathrm{PBS}$ 固定 $30 \mathrm{~min}$, PBS 漂洗后, 乙醇系列脱水, 醋酸异戊酯置换, $\mathrm{CO}_{2}$ 临界点干燥, 不经任何染色, 用 $\mathrm{CX}-100$ 透射 电镜观察 ${ }^{[9]}$.

4. DGD 包埋-去包埋制片，原生质体经上述选择性抽提后的样品, 在 $2.5 \%$ 成二醴一 LSK 溶液中固定 $40 \mathrm{~min}, 1 \% \mathrm{OsD}_{4}-\mathrm{PBS}$ 中继续固定 $60 \mathrm{~min}, \mathrm{PBS}$ 漂洗后乙醇系列脱水,正丁 醇置换后, DGD 609 包埋 3h. 然后在 LKB 切片机上切片, 用铜网捞取金色切片和紫色切 片, 正丁醇浸泡溶去 DGD, 经乙醇系列脱水, 醋酸异戊酯置换, $\mathrm{CO}_{2}$ 临界点干燥后, 不经染色 用 CX-100 透射电镜观察 ${ }^{[10]}$.

5. 免度印遮 经上述选择性抽提后的样品, 溶在 SDS 样品缓冲液中, 根据 Laemmli 的方法 ${ }^{[20]}$, 在稳压的条件下 (浓缩胶 $60 \mathrm{~V}$, 分离胶 $120 \mathrm{~V}$ ) 进行 SDS-PAGE 原电泳, 然后依照 Towbin 的方法 ${ }^{[1]}$, 转移到硝酸纤维滤膜上, 经 3\% BSA 封闭非特异性位点后, 与动物角蛋白 抗体 AF, 在 $37^{\circ} \mathrm{C}$ 躬育 $1-2 \mathrm{~h}$, 同 1:1000 稀释的碱性磷酸酶标记的羊抗鼠 IgG 抗体结台, 漂 洗后同 NBT、BCIP 底物进行显色反应.

角蛋白抗体 AF，购自重庆医科大学, 是鼠抗人的角蛋白抗体, 使用时 1:500稀释.

\section{二、结果和讨论}

韽豆根尖细胞经选择性抽提后, 无论在整装制片还是 DGD 包埋切片上都呈现精细的类 似动物细胞的中间纤维网架体系, 它们同动物细胞中间纤维一样, 对非离子去垢剂和高盐溶液 的处理有很大的稳定性. 同时我们选用免疫印迹技术对中间纤维进行定性分析, 证明其含有 二种不同分子量的类角蛋白成分. 现分别叙述如下:

\section{1. 类中间纤维的形态与结构}

（1）从致豆原生质体的整装制样可观察到中间纤维交织成网状, 在胞质内分布不均匀, 其 单纤维直径多为 $10 \mathrm{~nm}$, 但也有少量较粗的纤维存在, 这显然是由单丝聚集成束而形成的(图 1),同时还可见没有完全被抽提掉的变性细胞结构粘在纤维网上.

（2）在 DGD 包埋制片的样品上, 可观察到更为精细的中间纤维网状结构, 单丝直径基 
本为 $10 \mathrm{~nm}$, 从图 2 可以显示这些纤维网织成环, 存留出圆形“空洞”, 我们认为这可能是原液 泡被抽提掉的位置, 由于用 Triton X-100 处理溶去了液泡膜, 液泡内的物质也随之流失, 因 而留下了这个“空洞”, 其间的少数几根纤维丝可能是由于在干燥过程中从周围的纤维上脱落 下来的.

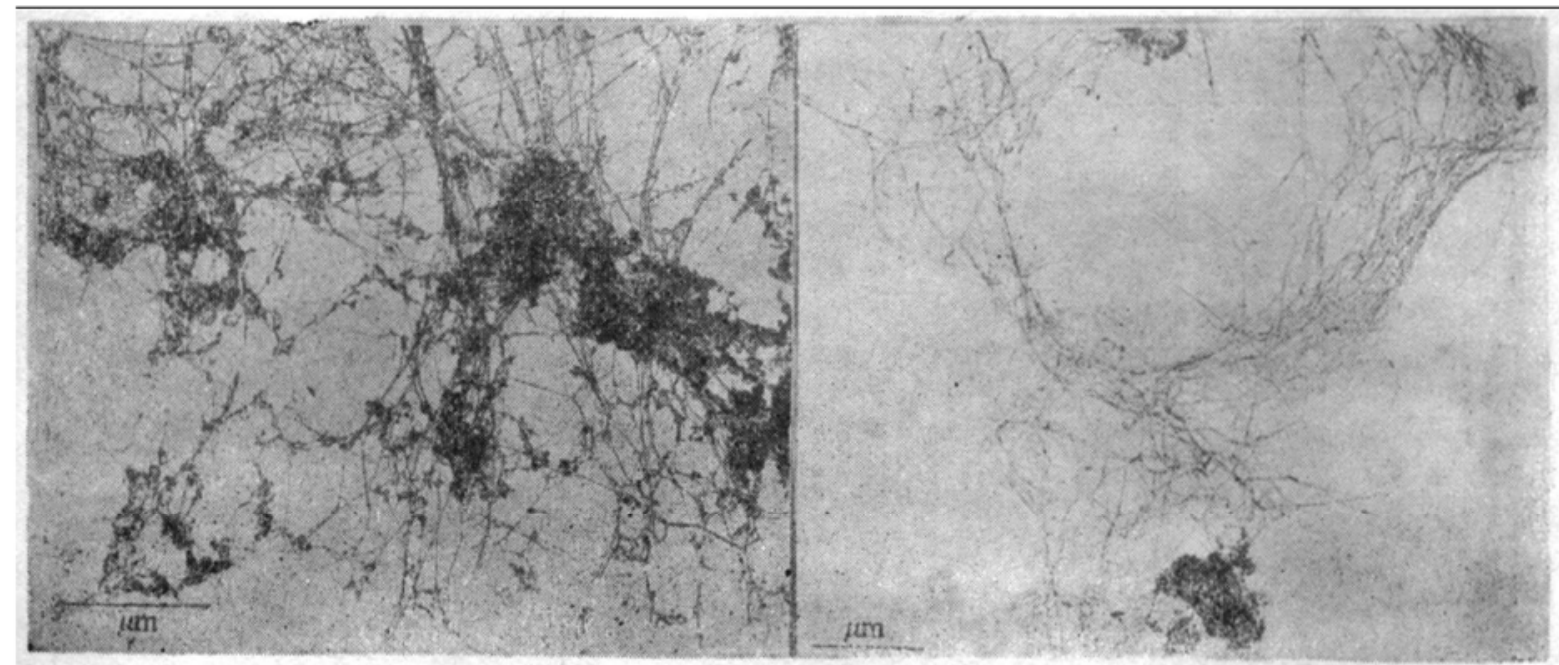

图 1 整㳖制备的晼豆根尖细胞

经过选择性抽提之后，盷质内显示出单丝直径 10 nn 的类角䍂白中间纤维构成的不均匀分布的致 密网络,未抽提尽的变性结构枮连在网络上
图 2 䤀豆根尖细胞经选择件抽提后的 DGD 包埋一去包埋切片

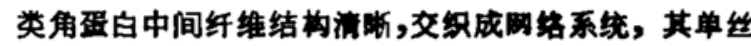
直径为 $100 \mathrm{~m}$

2. 兴中问纤维的成分分析 选择性抽提后得到的样品进行电泳分析表明, 样品中所含 的蛋白质种类很多, 用从动物细胞中得到的角蛋白抗体进行免疫检测, 发现其中有二条带与动 物细胞角蛋白抗体有阳性反应 (图 3), 其分子量分别为 52 和 $64 \mathrm{kD}$, 这与上述形态结构相印

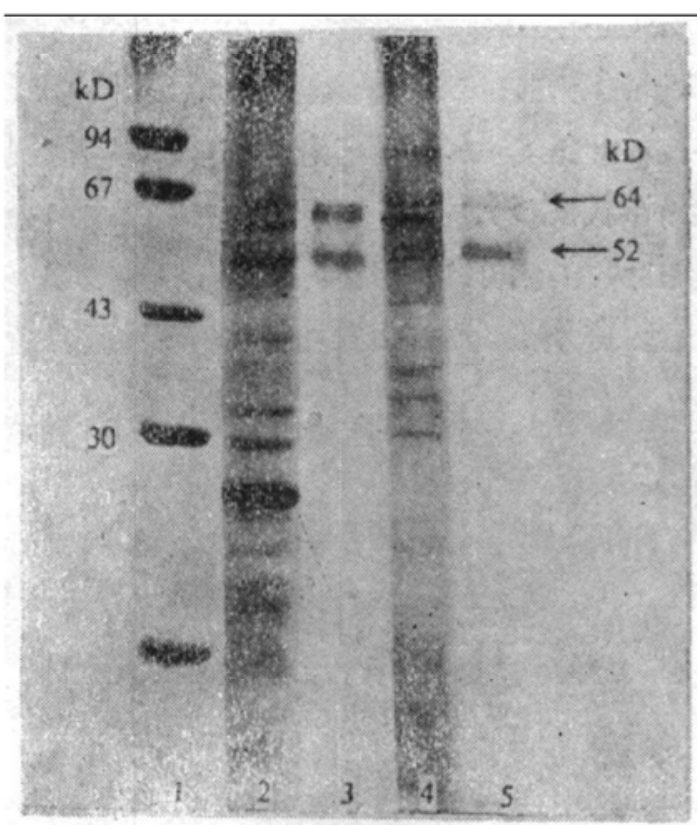

图 3 晼豆根尖细胞和白菜叶肉细胞选择姓抽提后得到的蛋白质汾的 SDS-PAGE 电永图谱和免度印迹结果

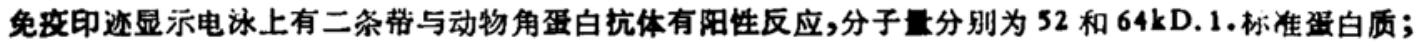
2. 白来叶肉细胞的 SDS-PAGE 电泳图谱;3. 白莱叶肉细胸的免度印迹图徣; 4. 䎸豆根尖细胞的 SDS-

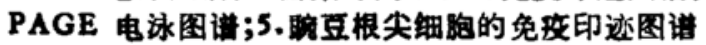


证, 更进一步证明在植物细胞中存在有与动物细胞角蛋白相类似的中间纤维.

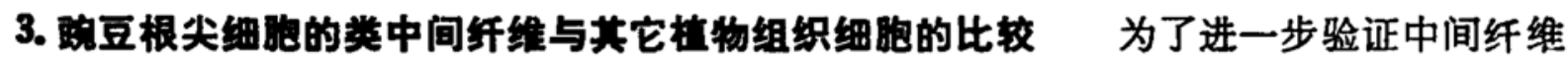
是否在植物细胞中普遍存在,我们同时用不同的植物组织做了对比实验.从白菜叶肉细胞的整 装制样中, 我们观察到了与㽬豆根尖细胞相同的结果(图 4), 直径 $10 \mathrm{~nm}$ 的中间纤维单丝在胞 质内构成精细的网络系统, 其致密程度较根尖细胞大. 免疫印迹的结果也证明在白菜、蚕豆叶 肉细胞和黄豆、寔豆根尖细胞中也存在有相同的䡗白质成分, 分子量均为 52 和 $64 \mathrm{kD}$ (图 3、5).

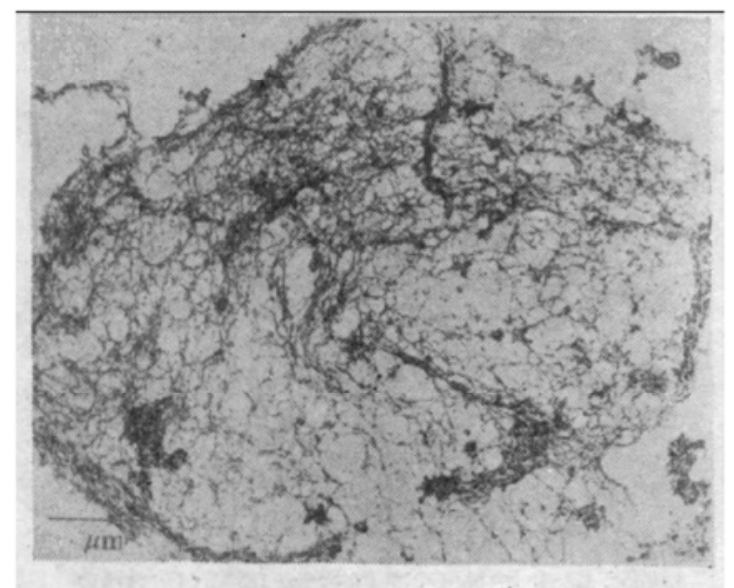

图4 白菜叶肉细胞的整装制片 速择性抽提后类角虽白中间纤维结合成网状, 不 均匀地分布在跑质区域内

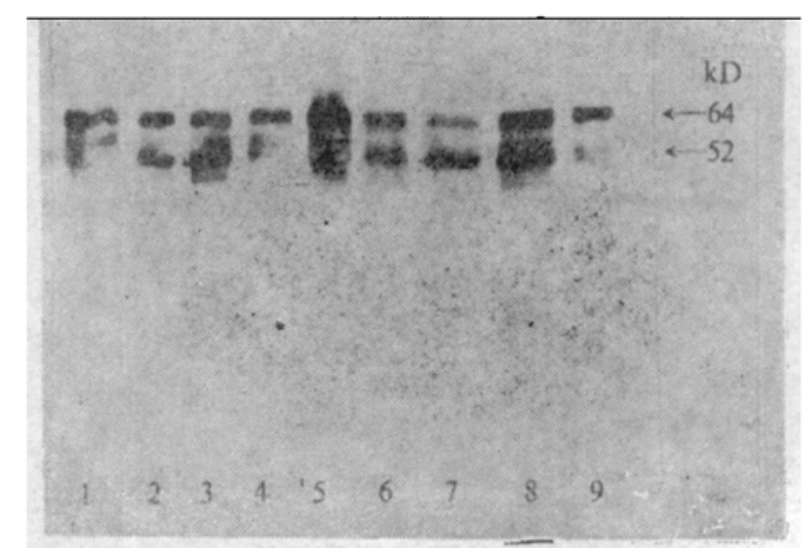

图 5 不同植物的根尖细胞与叶肉细胞分别用 液氮低温研磨裂解胞壁 $(1-5)$ 与酶解胞壁 (6-9) 后经选择性抽提的蛋白质成分的免痃印迹检测 它们均对动物角蛋白抗体有阳性反应,分别显示出 52 与 $64 \mathrm{kD}$ 两种分子量的盈白质,结果平行一致,说明植物细 胞的类角蛋白没有明显的种与组织的特开性. 图中自左 至右分别为 1 . 帵豆根尖细胞; 2 . 程豆根尖细胸;3.白莱叶

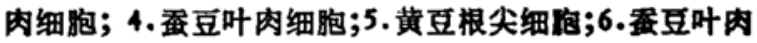

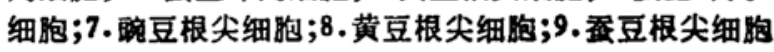

为了检证上述结果的稳定性和方法学的可靠性,我们又用液氮研磨破壁,冷 CSK 溶液提 取后选择性抽提的方法得到中间纤维样品, 对它进行免疫印迹检测的结果与上述敳豆根尖细 胞抽提的结果比较,结果完全相同(图 5).

根据上述结果,我们有理由认为中间纤维类角蛋白在植物细胞中广泛存在,不仅叶肉细胞 中有, 植物根尖细胞中也有, 其蛋白质成分没有表现出种与组织的特异性, 用不同方法抽提都 可得到稳定而一致的结果.

致谢: 本文得到翟中和教授的指导.

\section{今 考文献}

[1] Dawson, P. J. et al., J. Cell Biol., 100 (1985), 1793-1798.

[2] Millex, C. et al., Biochem. Soc. Trens., 13 (1985), 960-961.

[3] Powell, A. J. et al., J. Cell Sci., 56 (1982), 319-335.

[4] Goodbody, K. C. et al., J. Cell Sci., 93 (1989), 427-438.

[5] 苏菲、和中电子显微学报, $1988,3: 3$.

[6] 苏菲、蜀中和,中国科学。 B 辑, 1990,3: 267-270.

[7] Fey, E. G. et al., J. Cell Biol., 102 (1986), 1654-1665.

[8] 翼中和等, 中国科学, B 辑, 1987,10: 1076-1084.

[9] Capco, D. G. et al., J. Cell Biol., 98 (1984), 1878-1885.

[10] Laemmli, U. K. et al., Nature (London), 227 (1970), 680-685.

[11] Towbin, H. et al., Proc. Natl. Acad. Sci., 76 (1979), 4350-4354. 